\section{(1) \\ CrossMark}

\title{
Selection criteria for intensive care unit referral of lung cancer patients: a pilot study
}

\author{
Anne-Claire Toffart ${ }^{1,2}$, Carola Alegria Pizarro ${ }^{3}$, Carole Schwebel ${ }^{4,5}$, \\ Linda Sakhri ${ }^{2}$, Clemence Minet ${ }^{4}$, Michaël Duruisseaux ${ }^{2,6}$, Elie Azoulay ${ }^{7}$, \\ Denis Moro-Sibilot ${ }^{1,2}$ and Jean-François Timsit ${ }^{1,8,9,10}$
}

\begin{abstract}
Affiliations: 'Grenoble 1 University, U 823, A. Bonniot Institute, J. Fourier University, Grenoble, France. ${ }^{2}$ Thoracic Oncology Unit, Pôle Thorax et Vaisseaux, Centre Hospitalier Universitaire A. Michallon, Grenoble, France. ${ }^{3}$ Traumatology-Orthopedic Unit, Pôle Tête et Cou, Centre Hospitalier Universitaire A. Michallon, Grenoble, France. "Medical Intensive Care Unit, Pôle Urgences Médecine Aiguë, Centre Hospitalier Universitaire A. Michallon, Grenoble, France. ${ }^{5}$ Grenoble $1 \mathrm{~J}$. Fourier University, Laboratoires des Pharmaceutiques Biocliniques, U 1039, Grenoble, France. ${ }^{6}$ Equipe de Recherche 2, "Interactions des cellules tumorales et leur environnement et résistance aux agents anticancéreux", GRC Université Paris 6 Pierre et Marie Curie Theranoscan, Hôpital Tenon - APHP, Paris, France. ${ }^{7}$ Intensive Care Unit, Hôpital Saint Louis, Paris, France. ${ }^{8}$ INSERM, IAME, UMR 1137, Paris, France. ${ }^{9}$ Paris Diderot University, IAME, UMR 1137, Sorbonne Paris Cité, Paris, France. ${ }^{10}$ AP-HP, Hôpital Bichat, Service de Réanimation Médicale et Infectieuse,
\end{abstract} Paris, France.

Correspondence: Anne-Claire Toffart, UM Oncologie Thoracique, Pôle Thorax et Vaisseaux, Centre Hospitalier Universitaire A. Michallon, BP217, 38043 Grenoble Cedex 9, France. E-mail: AToffartQchu-grenoble.fr

ABSTRACT The decision-making process for the intensity of care delivered to patients with lung cancer and organ failure is poorly understood, and does not always involve intensivists. Our objective was to describe the potential suitability for intensive care unit (ICU) referral of lung cancer in-patients with organ failures.

We prospectively included consecutive lung cancer patients with failure of at least one organ admitted to the teaching hospital in Grenoble, France, between December 2010 and October 2012.

Of 140 patients, 121 (86\%) were evaluated by an oncologist and 49 (35\%) were referred for ICU admission, with subsequent admission for 36 (73\%) out of those 49 . Factors independently associated with ICU referral were performance status $\leqslant 2$ (OR 10.07, 95\% CI 3.85-26.32), nonprogressive malignancy (OR 7.00, 95\% CI 2.24-21.80), and no explicit refusal of ICU admission by the patient and/or family (OR 7.95, 95\% CI 2.39-26.37). Factors independently associated with ICU admission were the initial ward being other than the lung cancer unit (OR 6.02, 95\% CI 1.11-32.80) and an available medical ICU bed (OR 8.19, 95\% CI 1.48-45.35).

Only one-third of lung cancer patients with organ failures were referred for ICU admission. The decision not to consider ICU admission was often taken by a non-intensivist, with advice from an oncologist rather than an intensivist.

@ERSpublications

The decision not to consider ICU admission of lung cancer patients is often taken by a nonintensivist physician http://ow.ly/C4bNB

For editorial comments see Eur Respir J 2015; 45: 308-309 [DOI: 10.1183/09031936.00198714]

This article has supplementary material available from erj.ersjournals.com

Received: June 272014 | Accepted after revision: Sept 172014 | First published online: Oct 162014

Clinical trial: This study is registered at www.clinicaltrials.gov with identifier number NCT00222404.

Support statement: This project received funding from the publicly funded, non-profit organisation Cancéropole Lyon Auvergne Rhône-Alpes.

Conflict of interest: Disclosures can be found alongside the online version of this article at erj.ersjournals.com

Copyright $\odot$ ERS 2015 


\section{Introduction}

The mortality rate among lung cancer patients admitted to the intensive care unit (ICU) has dropped from $85 \%$ in 1986 [1] to $22-47 \%$ in 2005 [2-6]. These survival gains are ascribable to improvements in anticancer drugs [7-9] and intensive care [10], as well as to changes in criteria for ICU admission [11]. Selection of the intensity of care delivered to lung cancer patients with organ failure is based on the medical history [2-6], opinion of the oncologist and intensivist, and wishes of the patient and family. Available studies are retrospective [2-6] or confined to patients referred for ICU admission [12]. No published study has investigated the criteria for ICU referral of lung cancer patients or the impact of ICU referral and admission on patient outcomes.

Our objective here was to describe the potential suitability for ICU referral of lung cancer in-patients. We conducted a prospective, observational cohort study of lung cancer in-patients with organ failure.

\section{Materials and methods}

Study design and patients

We included all patients with lung cancer and failure of at least one organ admitted to any of the departments of the teaching hospital in Grenoble, France, between December 1, 2010, and October 31, 2012. Organ failure development during the postoperative period was an exclusion criterion.

Our primary objective was to identify factors associated with referral for ICU admission. The secondary objectives were to identify factors associated with ICU admission and to evaluate the consequences of intensity of care on patient outcomes, namely, mortality, psychological distress and hospital experience. This study was registered at www.ClinicalTrials.gov with the identifier NCT00222404.

The definition of organ failure used for the study is provided in online supplementary table S1. The appropriateness of ICU admission was discussed with the thoracic oncologist in charge of the patient, if available, and with the intensivist if contacted by the first physician in charge of the patient. Intensity of care decisions were based on the patient's performance status, lung cancer characteristics and organ failure, and the wishes of the patient and family, particularly regarding ICU admission. The reasons for choosing the initial intensity of care were recorded. Patients were followed prospectively in their hospital department. After 3 months, patients without cognitive disorders or terminal palliative care underwent an interview with a psychologist to evaluate their experience during their acute illness.

\section{Data collection}

The following data were collected prospectively: histological type of cancer, cancer status (remission, newly diagnosed or progression/recurrence), cancer spread (TNM classification 13), anticancer treatment, performance status (Eastern Cooperative Oncology Group Performance Status (ECOG-PS) 14) and Logistic Organ Dysfunction score to evaluate the acute disease. Prospective data on the circumstances of the decision-making process for determining ICU referral were as follows: hospital department, decision made during the day or off-hours, advice from specialists if any, number of beds available in the medical ICU at the time of organ failure development and reason for ICU admission refusal where relevant.

After 3 months, we recorded the ECOG-PS and the patients were assessed using the Hospital Anxiety and Depression Scale (HADS) [15], with sub-score cut-offs $\geqslant 11$ defining anxiety disorders or depression; the revised Impact of Event Scale (IES-R), with a total score $>25$ defining moderate-to-severe post-traumatic stress disorder (PTSD) [16, 17]; and the 36-item Short Form (SF-36), with higher scores on the Physical Component Summary (PCS) and Mental Component Summary (MCS) subscales indicating better health-related quality of life. These instruments were completed in the presence of a psychologist, both to alleviate patient anxiety related to the evaluation and to ensure that the patients fully understood all items.

\section{Statistical analysis}

Continuous variables are presented as median (interquartile range (IQR)) and categorical variables as $\mathrm{n}(\%)$. Associations between categorical variables were compared using the Chi-squared test or Fisher's exact test and those between continuous variables using the Wilcoxon test. No patient was lost to follow-up. Patients were followed until April 1, 2014.

To identify associations between patient characteristics and referral for ICU admission, ICU admission or 3-month mortality, we built univariate logistic regression models and computed the odds ratios with their 95\% confidence intervals. A preliminary choice between collinear variables was performed based on clinical relevance, accuracy of data entry and the Akaike Information Criterion [18]. Variables with $p<0.20$ were proposed to a stepwise procedure and were kept in the multiple logistic regression models when they yielded $\mathrm{p}$-values $\leqslant 0.05$ in the multivariate context. 
FIGURE 1 Flow chart. ICU: intensive care unit.

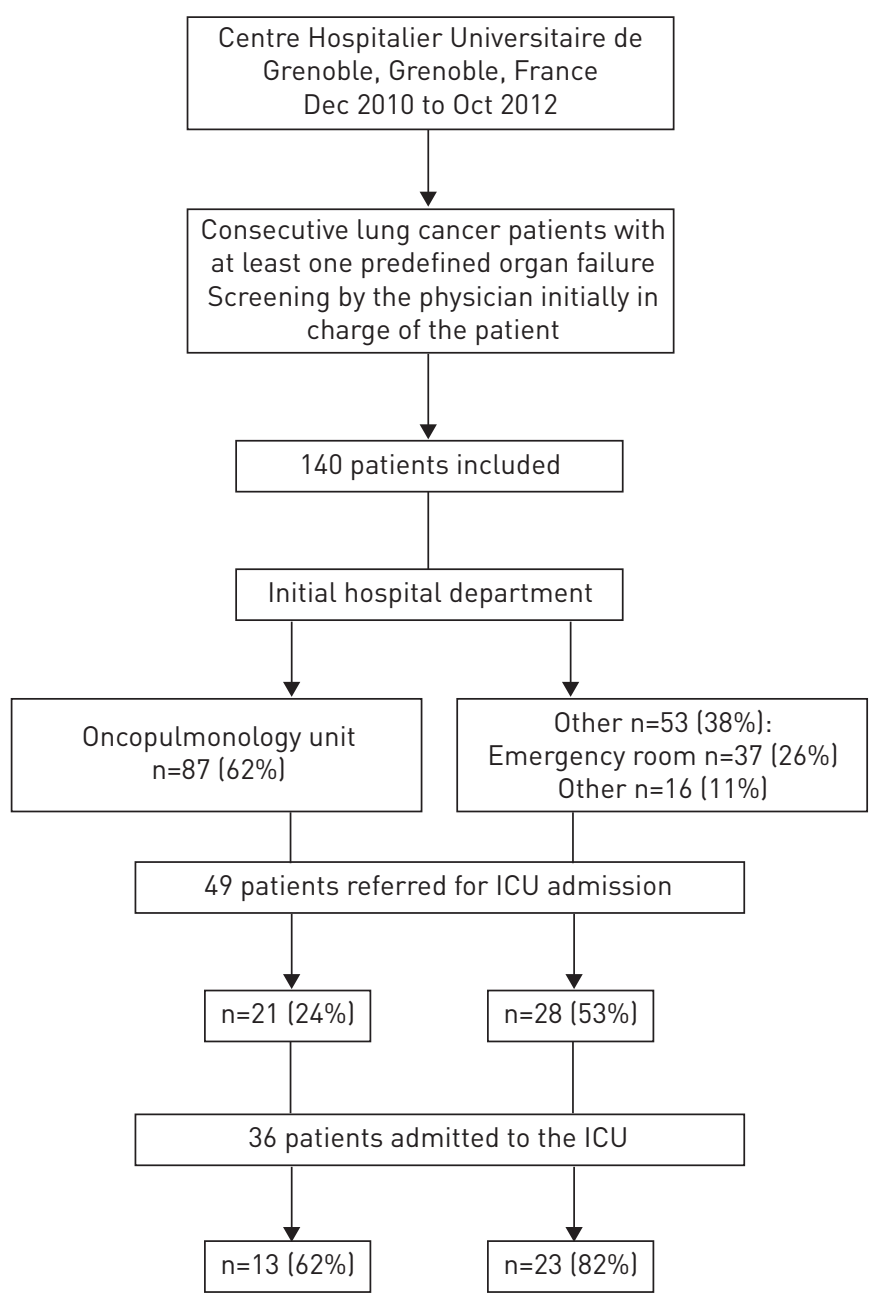

Kaplan-Meier plots of survival curves from organ failure onset were compared between groups using the log-rank test. The aforementioned method was used to select variables for a multivariate Cox model.

All tests were two-sided and p-values $<0.05$ were considered statistically significant. All statistical analyses were performed using SAS 9.3 (SAS Institute, Cary, NC, USA).

\section{Results}

\section{Patient characteristics}

We included 140 patients (fig. 1). During the study period, 550 patients were admitted to the emergency room and 1532 to other hospital departments for reasons other than cancer surgery. Table 1 lists the main patient characteristics and table 2 the characteristics of their critical illnesses. Among patients who developed organ failure, 82 (62\%) were referred from the lung cancer unit, 37 (26\%) from the emergency room and 16 (11\%) from other hospital departments.

Patient characteristics according to their initial department of admission are reported in table S2. Patients who developed organ failure in the lung cancer unit more often had an ECOG-PS >2 (OR 3.70, 95\% CI 1.80-7.61) than did patients who developed organ failure in other hospital departments. Patients in the lung cancer unit had a longer median time from cancer diagnosis to organ failure (median 7.1 months (IQR 1.5-20.7 months) versus 2.3 months (IQR 0.5-8.8 months), $\mathrm{p}=0.01$ ) and more often had progressive disease (OR 2.50, 95\% CI 1.18-5.32).

Of the 140 patients, 54 (39\%) said that they would agree to ICU admission should this option be offered to them and $49(35 \%)$ that they would refuse ICU admission; for the remaining 37 (26\%) patients, no information on patient wishes was available, and 20 (54\%) out of these 37 patients were not competent at the time they developed organ failure. Advice from the oncologist was obtained for 121 (86\%) patients and 49 (35\%) patients were referred for ICU admission, including 36 (73\%) out of 49 who were admitted to the ICU. 
TABLE 1 Patient characteristics according to referral for intensive care unit (ICU) admission

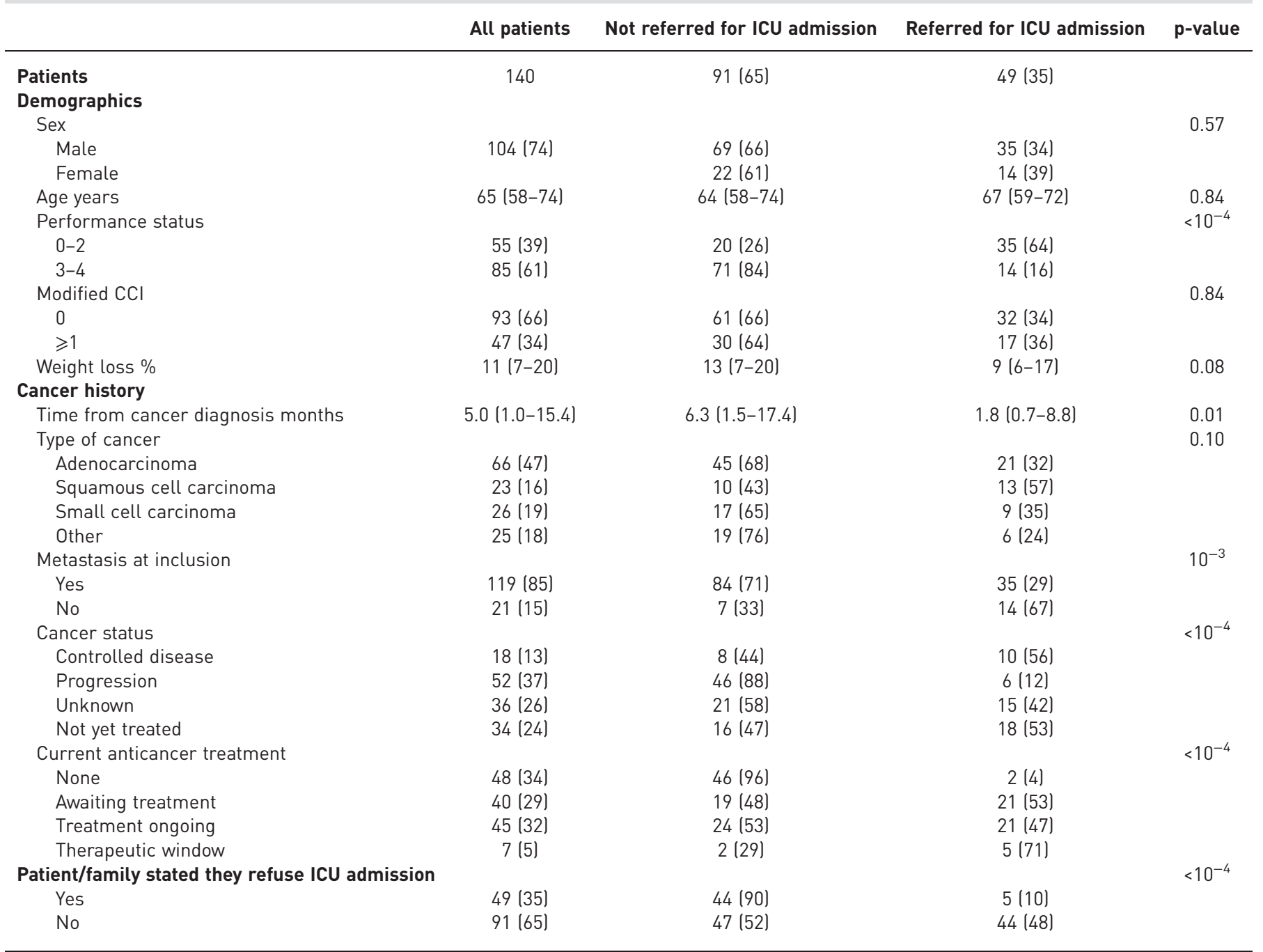

Data are presented as $\mathrm{n}, \mathrm{n}(\%)$ or median (interquartile range), unless otherwise stated. CCI: Charlson Comorbidity Index [19].

Of the eight patients for whom no bed was available in the hospital's medical ICU, seven were admitted to ICUs in other hospitals or to the same hospital's intermediate care unit; the remaining patient stayed in the emergency room.

Intensity of care was ICU admission in $36(26 \%)$ patients, maximal medical care without ICU admission in $52(37 \%)$ patients and palliative care in $52(37 \%)$ patients.

\section{Referral for ICU admission}

The main reason for not referring patients for ICU admission ( $\mathrm{n}=91,65 \%)$ was refusal by the patient and/ or family $(n=44,48 \%)$. The other main reasons given by the non-intensivists initially in charge of the patient (with several reasons per patient in some cases) were a decision to provide palliative care $(\mathrm{n}=31$, $34 \%)$, a bedridden patient $(n=27,30 \%)$, at least three different lines of chemotherapy $(n=16,18 \%)$, active brain metastasis $(\mathrm{n}=15,16 \%)$ and highly aggressive cancer $(\mathrm{n}=11,12 \%)$.

The univariate analysis results are reported in tables 1 and 2. Referral for ICU admission was more common when the physician in charge was not a lung cancer specialist $\left(\mathrm{p}=6 \times 10^{-4}\right)$. Factors independently associated with referral for ICU admission were ECOG-PS $\leqslant 2$ (OR 10.07, 95\% CI 3.85-26.32), nonprogressive malignancy (OR 7.00, 95\% CI 2.24-21.80), and absence of refusal stated explicitly by the patient and/or family (OR 7.95, 95\% CI 2.39-26.37). 
TABLE 2 Characteristics of the acute illnesses according to referral for intensive care unit (ICU) admission

All patients Not referred for ICU admission Referred for ICU admission p-value

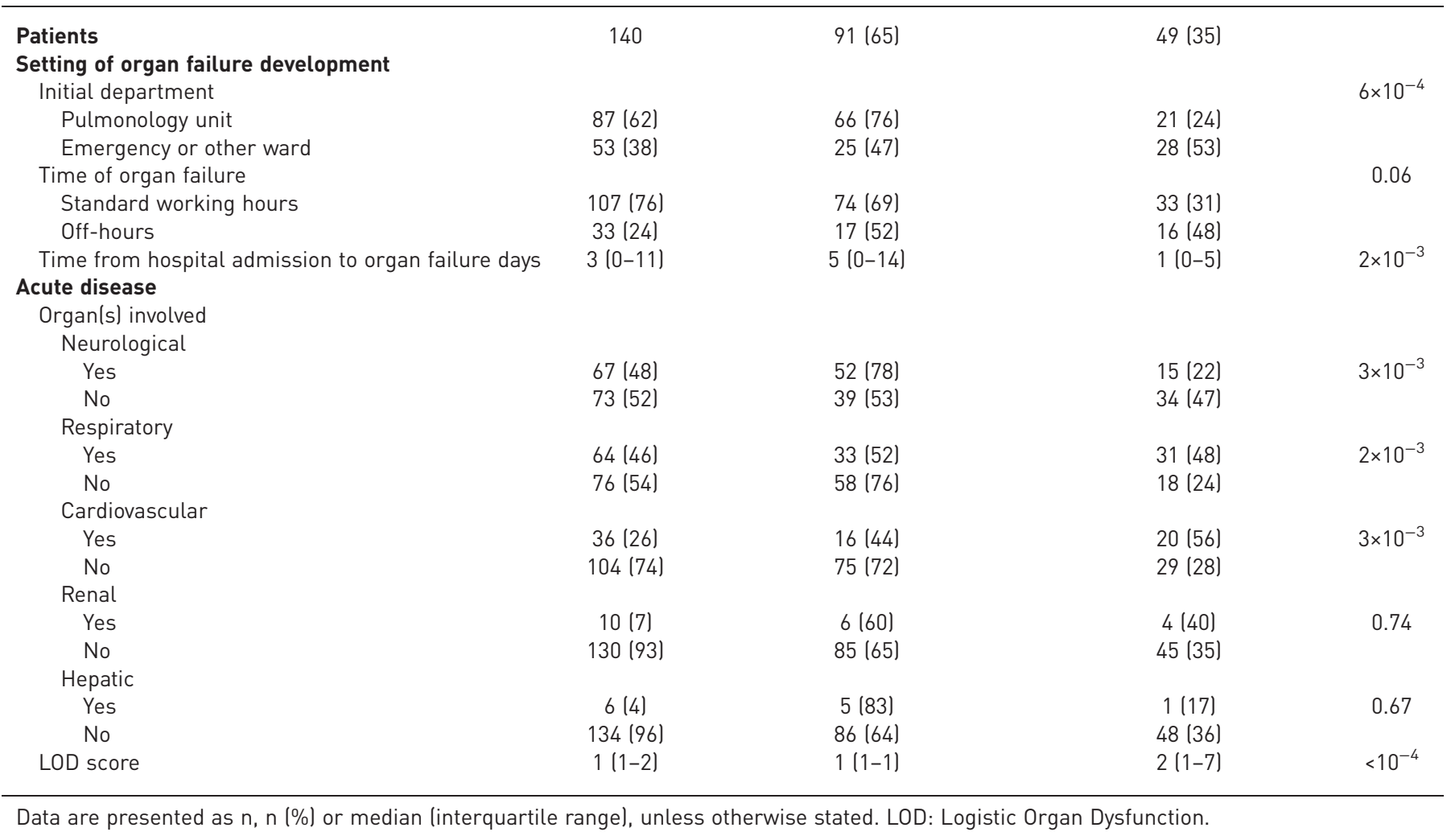

\section{ICU admission}

Tables 3 and 4 report the characteristics of the patients referred for ICU admission according to whether they were admitted. The main reasons for not admitting a patient $(n=13)$ given by the intensivists were excessive tumour spread (six (46\%) out of 13 patients) and excessive severity of the acute illness (five (38\%) out of 13). Among patients admitted to the ICU, 21 received vasopressors, 21 invasive mechanical ventilation and seven noninvasive ventilation.

In the patients referred for ICU admission, none of the demographic variables or critical-illness characteristics was significantly associated with ICU admission by univariate analysis. ICU admission decisions were similar during working days and during nights and weekends. By multivariate analysis, factors associated with ICU admission were initial admission to a department other than the lung cancer unit (OR 6.02, 95\% CI 1.1132.80 ) and an available bed in the hospital's medical ICU (OR 8.19, 95\% CI 1.48-45.35).

After initial refusal, two patients were subsequently admitted to the ICU but died during the ICU stay.

\section{Survival rates and prognostic factors}

Among patients initially admitted to the ICU, 18 (50\%) died in the ICU, and six others died between ICU discharge and hospital discharge. In-hospital mortality was 63\% $(n=33)$ among patients who received maximal medical care without ICU admission and $94 \%(n=49)$ among those who received palliative care. In the overall population, median survival was 5 days (IQR 1-26 days, range 0-981 days). Figure 2 shows survival according to referral for ICU admission and to ICU admission. Table 5 reports the results of the univariate analysis. Independent predictors of death were poor chronic health status, neurological or respiratory failure, and refusal of ICU admission by the patient or relatives. Interestingly, by multivariate analysis (table 5), ICU admission was not associated with survival. Only performance status was independently associated with 3-month survival (data not shown).

Among hospital survivors ( $\mathrm{n}=34,24 \%$ ), median survival after hospital discharge was 337 days (IQR 58-711 days) in patients admitted to the ICU ( $\mathrm{n}=12), 58$ days (IQR 9-118 days) in those who received maximal medical care $(n=19)$ and 29 days (IQR 12-349 days) in those who received palliative care $(n=3)$. 
TABLE 3 Characteristics of patients referred for intensive care unit (ICU) admission according to whether they were admitted

Admitted to the ICU

Patients

Demographics

Sex

Male

Female

Age years

Performance status

$0-2$

3-4

Modified CCl

0

$\geqslant 1$

Weight loss \%

Cancer history

Time from cancer diagnosis

Type of cancer

Adenocarcinoma

Squamous cell carcinoma

Small cell carcinoma

Other

Metastasis at inclusion

Yes

No

Cancer status

Controlled disease

Progression

Unknown

Not yet treated

Current anticancer treatment

None

Awaiting treatment

Treatment ongoing

Therapeutic window

Patient/family explicitly refused ICU admission

Yes

No
36 (73)

Not admitted to the ICU

p-value

13 (27)

$$
25 \text { (71) }
$$

$11(79)$

67 (59-74)

27 (77)

$9(64)$

$22(69)$

$14(82)$

10 (4-17)

$1(0-6)$

18 (86)

9 (69)

5 (56)

4 (67)

24 (69)

12 (86)

7 (70)

4 (67)

10 (67)

15 (83)

1 (50)

17 (81)

14 (67)

4 (80)

$3(60)$

33 (75)
10 (29)

3 (21)

64 (57-70)

8 (23)

5 (36)

10 (32)

3 (18)

9 (6-13)

9 (1-19)

3 (14)

4 (31)

4 (44)

2 (33)

11 (31)

2 (14)

3 (30)

2 (33)

5 (33)

3 (17)

1 (50)

4 (19)

7 (33)

1 (20)

2 (40)

11 (25)
0.46

0.11

0.30

0.73

0.48

Data are presented as $\mathrm{n}(\%)$ or median (interquartile range), unless otherwise stated. CCI: Charlson Comorbidity Index [19].

Only two patients with ECOG-PS $>2$ at baseline were still alive after 3 months. Both had progressive malignancies and neither was admitted to the ICU. After 3 months, their ECOG-PS was 4 and they were in palliative care without having received further anticancer treatment.

\section{Evaluation at 3 months}

At 3 months, only 19 (14\%) patients were still alive, including 10 who underwent a psychological evaluation; one of these patients was not assessed using the HADS, IES-R of SF-36. The reasons for not undergoing this evaluation in the other nine patients were end-of-life (EOL) setting $(n=3)$, cognitive disorders $(n=3)$, patient refusal $(n=2)$ and loss to follow-up $(n=1)$.

HADS results showed anxiety in one patient and depression in another; both patients had been admitted to the ICU. IES-R results indicated PTSD in five patients; the four patients without PTSD had no anxiety or depression. All 10 evaluated patients had suffered a threatening experience at the psychological and physical levels, and had difficulty coping with the loss of their previous healthy condition. They overinvested their daily activities, which were often limited by physical exhaustion. The mean \pm SD SF-36

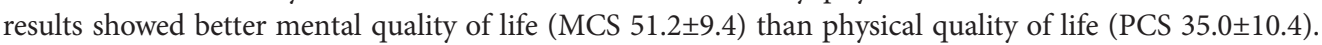

\section{Discussion}

We report the results of a 2-year, prospective, hospital-wide study designed to evaluate the potential suitability for ICU referral of 140 consecutive patients with lung cancer and failure of one or more organs. 
TABLE 4 Characteristics of acute illnesses in patients referred for intensive care unit (ICU) admission according to whether they were admitted

Admitted to the ICU

Yes

Acute disease

Organ(s) involved

Neurological

Yes

No

Respiratory

Yes

No

Cardiovascular

Yes

No

Renal

Yes

No

Hepatic

Yes

No

LOD score
$36(73)$

$13(62)$

$23(82)$

22 (67)

$14(88)$

$0(0-4)$

8 (53)

28 (82)

Not admitted to the ICU

p-value

13 (27)

8 (38)

5 (18)

11 (33)

2 (13)

$4(0-11)$

$7(47)$

6 (18)

$10(67)$

5 (33)

$8(24)$

6 (19)

7 (39)

$11(61)$

$3(15)$

10 (34)

2 (50)

$11(24)$

0 (0)

13 (27)

$1(1-2)$

Data are presented as $\mathrm{n}(\%)$ or median (interquartile range), unless otherwise stated. If no bed was available in the hospital's medical ICU, patients could be admitted to another ICU or to an intermediate-care unit. LOD: Logistic Organ Dysfunction.

We found that $65 \%$ of patients were not referred to the ICU, i.e. that intensive care was often withheld by physicians who were not intensivists. Patient-related factors associated with absence of referral for ICU admission were poor ECOG-PS, progressive malignancy, and explicit refusal of ICU admission by the patient and/or family; structure-related factors were initial admission to the lung cancer unit and lack of available beds in the medical ICU.

Strengths of our study include the prospective design and the evaluation of the strategy chosen by the first physician in charge of the patient. A single previous prospective study [12] focused on the decision-making process for ICU admission but this study was confined to patients referred to the ICU and therefore missed the patients for whom the oncologists decided that ICU admission was not appropriate. We also evaluated psychological distress using both standard scales and a psychologist's evaluation in 3-month survivors. The single-centre patient recruitment limits the external applicability of our results. Furthermore, the process of care was probably organised toward cancer patients, as considerable efforts have been made in our institution over the years to improve communication between intensivists and lung cancer specialists regarding the early evaluation and triage of patients. We did not record the details of the discussions regarding treatment-limitation decisions.

Importantly, in our hospital, only 35\% of lung cancer patients with organ failure were referred for ICU admission overall, and this proportion was only $24 \%$ among patients in the lung cancer unit. Thus, lung cancer specialists had a strong tendency to consider that ICU admission was inappropriate. The main reasons were poor general status of the patient or advanced cancer; another reason identified in nearly half the cases was explicit refusal of ICU admission by the patient and/or family. In a study of 1231 patients with stage IV lung or colorectal cancer [20], 47\% of patients received at least one aggressive therapeutic intervention within 30 days before death, including ICU admission (6\% of patients), and patient- and surrogate-reported EOL discussions were significantly associated with EOL care. These data emphasise the importance of holding EOL 


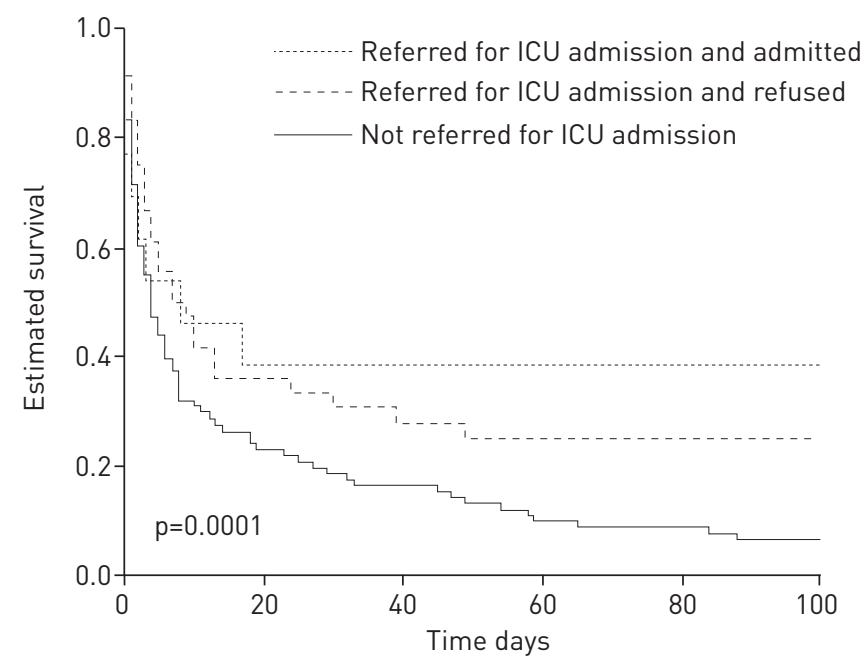

\begin{tabular}{|l|c|c|c|c|c|c|c|c|}
\hline Time days & $\mathbf{0}$ & $\mathbf{5}$ & $\mathbf{1 0}$ & $\mathbf{2 0}$ & $\mathbf{4 0}$ & $\mathbf{6 0}$ & $\mathbf{8 0}$ & $\mathbf{1 0 0}$ \\
\hline At risk n & 140 & 73 & 53 & 40 & 29 & 29 & 21 & 19 \\
\hline $\begin{array}{l}\text { Referred and } \\
\text { admitted }\end{array}$ & 36 & 23 & 18 & 14 & 10 & 10 & 9 & 9 \\
\hline $\begin{array}{l}\text { Referred and } \\
\text { refused }\end{array}$ & 13 & 7 & 6 & 5 & 4 & 4 & 4 & 4 \\
\hline Not referred & 91 & 43 & 29 & 21 & 15 & 9 & 8 & 6 \\
\hline
\end{tabular}

FIGURE 2 Kaplan-Meier survival estimate according to referral for intensive care unit (ICU) admission.

discussions attended by physicians and patients. The reasons given for not referring patients to the ICU were straightforward. The main issue is whether intensive care would have improved survival and quality of life had the oncologists (and patients/relatives) been in favour of ICU admission.

Among patients referred for ICU admission, factors associated with ICU admission were initial admission to a department other than the lung cancer unit and an available bed in the medical ICU. In another study [12], among patients with haematological or solid malignancies referred for ICU admission, remission of the malignancy was associated with ICU admission, whereas poor chronic health status and solid malignancy were associated with refusal of ICU admission. In our study, these factors were associated with referral for ICU admission but were not significantly associated with ICU admission among referred patients.

TABLE 5 Univariate and multivariate analyses of factors associated with mortality

Factor

\section{Univariate analysis}

\section{HR $(95 \% \mathrm{Cl})$}

$1.36(0.97-1.91)$
$1.28(0.87-1.89)$
$2.40(1.63-3.55)$
$1.21(0.85-1.72)$
$1.88(1.11-3.18)$
$1.49(1.04-2.14)$
$1.04(0.74-1.45)$
$1.67(1.17-2.37)$
$1.43(1.02-2.01)$
$0.82(0.56-1.22)$
$1.66(1.16-2.38)$
$1.61(1.13-2.30)$
$0.61(0.41-0.91)$

\subsection{8}

0.21

$<10^{-4}$

0.30

Modified $\mathrm{CCl} \geqslant 0$

Cancer history

Metastasis at inclusion

Progression

Cancer diagnosis $>5$ months earlier

Organ failure

Neurological

Respiratory

Cardiovascular

ICU refusal from patient/relatives

Referred by pulmonologist

ICU admission

HR: hazard ratio; ECOG-PS: Eastern Cooperative Oncology Group Performance Status; CCI: Charlson Comorbidity Index [19]; ICU: intensive care unit. 
In keeping with previous studies, factors associated with decreased survival were a poor ECOG-PS [5, 21$24]$ and respiratory failure $[4,25,26]$. Survival was very poor, in part due to the absence of anticancer treatment in one-third of patients. In patients not receiving anticancer treatment, the development of organ failure can indicate the beginning of the dying process. Survival of patients admitted to the ICU was worse than in previous studies $[2,3,5]$, whereas survival was good in patients discharged alive from the hospital, most notably after ICU admission.

All 10 patients evaluated by the psychologist reacted negatively to the stress associated with the acute event. The traumatic effect may have been related to the experience of impending death, although the acute event did not induce psychological trauma. Finally, the traumatic event was described by the patients as a reminder of the severity of the cancer and of the risks related to cancer progression and treatment. This last point constitutes a strong incentive to develop early supportive care and psychological support for cancer patients.

\section{Conclusions}

The prognosis of lung cancer with organ failure was very poor, particularly in patients whose ECOG-PS was $>2$. When organ failure developed, only $35 \%$ of patients with lung cancer were referred for ICU admission in our teaching hospital. Thus, the triage decision was often made without advice from intensivists. These results require confirmation by a multicentre study.

\section{Acknowledgements}

We thank A. Wolfe for English-language editing.

\section{References}

1 Ewer MS, Ali MK, Atta MS, et al. Outcome of lung cancer patients requiring mechanical ventilation for pulmonary failure. JAMA 1986; 256: 3364-3366.

2 Soares M, Darmon M, Salluh JI, et al. Prognosis of lung cancer patients with life-threatening complications. Chest 2007; 131: 840-846.

3 Adam AK, Soubani AO. Outcome and prognostic factors of lung cancer patients admitted to the medical intensive care unit. Eur Respir J 2008; 31: 47-53.

4 Roques S, Parrot A, Lavole A, et al. Six-month prognosis of patients with lung cancer admitted to the intensive care unit. Intensive Care Med 2009; 35: 2044-2050.

5 Toffart AC, Minet C, Raynard B, et al. Use of intensive care in patients with nonresectable lung cancer. Chest 2011; 139: 101-108.

6 Andrejak C, Terzi N, Thielen S, et al. Admission of advanced lung cancer patients to intensive care unit: a retrospective study of 76 patients. BMC Cancer 2011;11: 159 .

7 Sandler A, Gray R, Perry MC, et al. Paclitaxel-carboplatin alone or with bevacizumab for non-small-cell lung cancer. N Engl J Med 2006; 355: 2542-2550.

8 Maemondo M, Inoue A, Kobayashi K, et al. Gefitinib or chemotherapy for non-small-cell lung cancer with mutated EGFR. N Engl J Med 2010; 362: 2380-2388.

9 Shaw AT, Kim DW, Nakagawa K, et al. Crizotinib versus chemotherapy in advanced ALK-positive lung cancer. $N$ Engl J Med 2013; 368: 2385-2394.

10 Pene F, Percheron S, Lemiale V, et al. Temporal changes in management and outcome of septic shock in patients with malignancies in the intensive care unit. Crit Care Med 2008; 36: 690-696.

11 Azoulay E, Soares M, Darmon M, et al. Intensive care of the cancer patient: recent achievements and remaining challenges. Ann Intensive Care 2011; $1: 5$.

12 Thiery G, Azoulay E, Darmon M, et al. Outcome of cancer patients considered for intensive care unit admission: a hospital-wide prospective study. J Clin Oncol 2005; 23: 4406-4413.

13 Groome PA, Bolejack V, Crowley JJ, et al. The IASLC Lung Cancer Staging Project: validation of the proposals for revision of the $\mathrm{T}, \mathrm{N}$, and $\mathrm{M}$ descriptors and consequent stage groupings in the forthcoming (seventh) edition of the TNM classification of malignant tumours. J Thorac Oncol 2007; 2: 694-705.

14 Oken MM, Creech RH, Tormey DC, et al. Toxicity and response criteria of the Eastern Cooperative Oncology Group. Am J Clin Oncol 1982; 5: 649-655.

15 Zigmond AS, Snaith RP. The hospital anxiety and depression scale. Acta Psychiatr Scand 1983; 67: 361-370.

16 Horowitz M, Wilner N, Alvarez W. Impact of Event Scale: a measure of subjective stress. Psychosom Med 1979; 41: 209-218.

17 Weiss DS, Marmar CR The impact of event scale - revised. In: Wilson JP, Keane TM, eds. Assessing Psychological Trauma and PTSD. New York, Guilford Press, 1997; pp. 399-411.

18 Akaike H. A new look at the statistical model identification. IEEE Trans Automat Control 1974; 19: 716-723.

19 Quan H, Li B, Couris CM, et al. Updating and validating the Charlson comorbidity index and score for risk adjustment in hospital discharge abstracts using data from 6 countries. Am J Epidemiol 2011; 173: 676-682.

20 Mack JW, Cronin A, Keating NL, et al. Associations between end-of-life discussion characteristics and care received near death: a prospective cohort study. J Clin Oncol 2012; 30: 4387-4395.

21 Soares M, Caruso P, Silva E, et al. Characteristics and outcomes of patients with cancer requiring admission to intensive care units: a prospective multicenter study. Crit Care Med 2010; 38: 9-15.

22 Christodoulou C, Rizos M, Galani E, et al. Performance status (PS): a simple predictor of short-term outcome of cancer patients with solid tumors admitted to the intensive care unit (ICU). Anticancer Res 2007; 27: 2945-2948.

23 Lilenbaum RC, Cashy J, Hensing TA, et al. Prevalence of poor performance status in lung cancer patients: implications for research. J Thorac Oncol 2008; 3: 125-129. 
24 Moro-Sibilot D, Pluquet E, Zalcman G, et al. Quel traitement pour un patient de PS-2/3 ayant un cancer bronchique non à petites cellules (CBNPC) de stade IV? [What treatment for a patient of PS 2-3 with stage IV non-small cell lung cancer?]. Rev Mal Respir 2007; 24: 6S120-6S124.

25 Slatore CG, Cecere LM, Letourneau JL, et al. Intensive care unit outcomes among patients with lung cancer in the surveillance, epidemiology, and end results-medicare registry. J Clin Oncol 2012; 30: 1686-1691.

26 Reichner CA, Thompson JA, O'Brien S, et al. Outcome and code status of lung cancer patients admitted to the medical ICU. Chest 2006; 130: 719-723. 